2 Involvement of peptidyl-prolyl isomerase Pin1 in the inhibitory effect of

3 fluvastatin on endothelin-1-induced cardiomyocyte hypertrophy

5 Satoshi Sakai ${ }^{a^{*}}$, Nobutake Shimojo ${ }^{b^{*}}$, Taizo Kimura ${ }^{\mathrm{a}}$, Kazuko Tajiri ${ }^{\mathrm{a}}$, Hidekazu

6 Maruyama $^{a}$, Satoshi Homma ${ }^{a}$, Keisuke Kuga ${ }^{a}$, Taro Mizutani ${ }^{b}$, Kazutaka Aonuma ${ }^{a}$,

$7 \quad$ Takashi Miyauchi ${ }^{\mathrm{a}, \mathrm{c}}$

8 Divisions of ${ }^{\mathrm{a}}$ Cardiovascular Medicine and ${ }^{\mathrm{b}}$ Emergency and Critical Care Medicine,

9 Department of Clinical Medicine, Faculty of Medicine, and ' Life Science Center for

10 Tsukuba Advanced Research Alliance (TARA), University of Tsukuba, 1-1-1 Tennodai,

11 Tsukuba, Ibaraki 305-8575, Japan

12 A part of this work was presented at the $13^{\text {th }}$ International Conference on Endothelin

13 (held at University of Tsukuba, Tokyo Campus; Sep $8^{\text {th }}-11^{\text {th }}, 2013$ ).

\title{
14 Key words
}

15 Pin1, endothelin-1, statin, c-Jun, cardiomyocyte hypertrophy

16 Total words $5740 \quad 6$ Figures

17 *First two authors equally contributed to this study.

18 Corresponding: Satoshi Sakai, MD, PhD

19 Division of Cardiovascular Medicine, Department of Clinical Medicine, Faculty of

20 Medicine, University of Tsukuba

21 Address: 1-1-1 Tennodai, Tsukuba, Ibaraki 305-8575, Japan

22 E-mail: ssakai@md.tsukuba.ac.jp Phone: +81-29-853-3210 Fax: +81-29-853-3143 


\section{Abstract}

2 Aims: Cardiac hypertrophy is elicited by endothelin (ET)-1 as well as other

3 neurohumoral factors, hemodynamic overload, and oxidative stress; HMG-CoA

4 reductase inhibitors (statins) were shown to inhibit cardiac hypertrophy partly via the

5 anti-oxidative stress. One of the common intracellular pathways of them is the

6 phosphorylation cascade of MEK signaling. Pin1 specifically isomerizes the

7 phosphorylated protein with Ser/Thr-Pro bonds and regulates their activity through

8 conformational changes. There is no report whether the Pin1 activation contributes to

9 ET-1-induced cardiomyocyte hypertrophy and whether the Pin1 inactivation contributes

10 to the inhibitory effect of statins. The aim of this study was to reveal these questions.

11 Main methods: We assessed neonatal rat cardiomyocyte hypertrophy using ET-1 and

12 fluvastatin by the cell surface area, ANP mRNA expression, JNK and c-Jun

13 phosphorylation, and $\left[{ }^{3} \mathrm{H}\right]$-leucine incorporation. Key findings: Fluvastatin inhibited

14 ET-1-induced increase in the cell surface area, ANP expression, and $\left[{ }^{3} \mathrm{H}\right]$-leucine

15 incorporation; and it suppressed the signaling cascade from JNK to c-Jun. The

16 phosphorylated Pin1 level, an inactive form, was decreased by ET-1; however, it

17 became basal level by fluvastatin. Furthermore, Pin1 overexpression clearly elicited

18 cardiomyocyte hypertrophy, which was inhibited by fluvastatin. Significance: This is

19 the first report that ET-1-induced cardiomyocyte hypertrophy is mediated through the

20 Pin1 activation and that the inhibitory effect of fluvastatin on cardiomyocyte hypertrophy

21 would partly be attributed to the suppression of the Pin1 function. This study firstly

22 suggests that Pin1 determines the size of hypertrophied cardiomyocyte by regulating

23 the activity of phosphorylated molecules and that statins exert their pleiotropic effects

24 partly via Pin1 inactivation. 


\section{Introduction}

2 Cardiac hypertrophy is elicited by endothelin (ET)-1 (Suzuki et al., 1990; Yorikane et 3 al., 1993; Ito et al., 1997; Koleitis et al., 2013; Miyauchi and Goto, 2013) as well as other 4 neurohumoral factors, hemodynamic overload, and oxidative stress. One of the 5 common intracellular pathways of them is the phosphorylation cascade of MEK signaling. Peptidyl-prolyl cis-trans isomerase 1 (Pin1) is a highly conserved enzyme that isomerizes specific phosphorylated Ser/Thr-Pro bonds in certain proteins, inducing conformational changes (Lu et al., 1996). It has been reported that Pin1 has the binding activity to the pSer/Thr-Pro pocket of target protein and that Pin1 catalyzes such portion. Both of these activities are exerted when the Ser-16 residue of Pin1 is dephosphorylated; on the other hand, these activities are inhibited when the residue is phosphorylated (Lu et al., 2007). The family of proline-directed protein kinases containing a major regulatory phosphorylation motif (pSer/Thr-Pro) includes c-Jun, Akt, cyclin dependent kinases, Raf-1, SMAD2/SMAD3, etc., which play an important role in the regulation of cell proliferation, tumorigenesis, neurological disorders, and autoimmune and inflammatory diseases (Lee et al., 2011).

17 The endothelin (ET) system consists of two G protein coupled-receptors, ET type A receptor and ET type B receptor, and three endogenous ligands, ET-1, ET-2, and ET-3

19 (Davenport, 2002; Horinouchi et al., 2013; Koleitis et al., 2013; Miyauchi and Goto, 2013). ET-1, a potent vasoconstrictive peptide produced by endothelial cells

21 (Yanagisawa et al.,1988), is also produced by cardiomyocytes and contributes to the development of cardiac hypertrophy (Ito et al., 1991; Yorikane et al., 1993; Koleitis et al., 2013; Miyauchi and Goto, 2013). We reported that the production of ET-1 is markedly

24 increased in the failing hearts of rats with chronic heart failure (Sakai et al., 1996a; Sakai et al., 1996b) and that the enhancement of myocardial ET-1 contributes to the modulation of the cardiac function (Sakai et al., 1996a) and cardiac hypertrophy at the 
1 molecular level in the failing hearts (Sakai et al. 2000); moreover, chronic administration

2 of the $\mathrm{ET}_{\mathrm{A}}$ receptor antagonist $\mathrm{BQ}-123$ inhibits the cardiac remodeling and ameliorates

3 the cardiac function (Sakai et al., 1996b). In addition, the signaling cascades of the

4 mitogen activated protein kinase (MEK) family including extracellular signal-regulated

$5 \quad$ kinase (ERK) and c-Jun N-terminal kinase (JNK) are augmented by ET-1 in

6 cardiomyocyte hypertrophy (Yue et al., 2000; Irukayama-Tomobe et al., 2004; Shimojo

7 et al., 2006). Therefore, in the present study, we hypothesized that Pin 1 contributes to

8 the development of cardiomyocyte hypertrophy through the activation of phosphorylated

9 kinases of the MEK family by exerting its catalytic activity.

3-hydroxy-3-methylglutaryl coenzyme A (HMG-CoA) reductase inhibitors (statins)

11 are widely employed classes of cholesterol-lowering drugs that work through the inhibition of HMG-CoA reductase. Much evidence has demonstrated that statins reduce the cardiovascular risk to a greater extent than that expected based on the blood

14 cholesterol-lowering effect alone; those additional activities of statins are known as 15 pleiotropic effects including the suppression of inflammation and improvement of 16 endothelial dysfunction (Wierzbicki et al., 2003). We have reported that pitavastatin 17 ameliorates the severity of experimental autoimmune myocarditis through the inhibition of T-cell mediated autoimmunity (Tajiri et al., 2013). It was reported that statins interfere with the protein (iso)prenylation processes (Wierzbicki et al., 2003), which may be

20 involved in the regulation of several cellular mechanisms such as signal transduction 21 and cell proliferation and differentiation, etc. Previous studies have shown that statins 22 inhibit cardiomyocyte hypertrophy provoked by angiotensin II by blocking the Rho 23 kinase-mediated cyclin D1 activation (Morikawa-Futamatsu et al., 2006) and that an 24 antioxidant mechanism involving Rac1 inhibition (Takemoto et al., 2001). Such reports suggest that another unresolved mechanism for the pleiotropic effects of statins remains. 
1 However, there is no report whether the Pin1 activation is involved in cardiomyocyte

2 hypertrophy provoked by ET-1; therefore, we hypothesized that the activation of Pin1

3 activity would contribute to the ET-1-induced cardiomyocyte hypertrophy. In addition,

4 there is no report whether the inhibitory effect of statins on the ET-1-induced

5 cardiomyocyte hypertrophy is mediated through the modulation of the Pin1 activity. The

6 aim of this study was to reveal these questions.

\section{Materials and Methods}

9 Adenovirus vectors Human Pin1 cDNA cloned from 293A cells by PCR using the

10 following primers, (Forward) 5'-CACCATGGCGGACGAGGAGAAGCT-3' and (Reverse)

11 5'-CTCAGTGCGGAGGATGATGTGGATG-3', was ligated to the pENTR-TOPO plasmid 12 as an entry clone; cDNA was transferred to the pAd/CMV/V5-DEST Gateway vector by 13 an LR recombination (Life Technologies, Carlsbad, CA). The cDNA for human Pin1 was 14 designed to add the V5 tag at C-termianl of the entire Pin1 fusion protein. The 15 adenovirus vector plasmid was digested by Pac I, transfected to $293 \mathrm{~A}$ cells and 16 amplified, and finally purified by a Vivaspin column system (Sartorius Stadium 17 Biotechnology, Goettingen, Germany). As a control, an adenovirus vector of LacZ was used. Adenovirus for an enhanced green fluorescent protein (EGFP) was used to

19 visualize the morphology of the cardiomyocytes and to take photographs.

20 Cardiomyocyte culture Neonatal rat cardiomyocytes were isolated from 2- to

21 3-day-old Sprague-Dawley rats, as described previously (Shimojo et al., 2007; Sakai et

22 al., 2012), and were incubated on fibronectin-coated dishes in DMEM-Ham's F-12

23 medium (Wako) supplemented with $0.1 \%$ fatty acid-free bovine serum albumin (BSA)

24 (Sigma, St. Louis, $\mathrm{MO}$ ) in $95 \%$ air- $5 \% \mathrm{CO}_{2}$. The cells were cultured for 2 days after the 25 differential adhesion and then used for further experiments. The animal experiment for 
1 cardiomyocyte isolation was carried out in a humane manner after we received approval

2 from the Institutional Animal Experiment Committee of University of Tsukuba and were

3 in accordance with the Regulation for Animal Experiments in our university.

4 Study Protocol Cardiomyocytes were pretreated with a vehicle or fluvastatin (0.1-10

$5 \mu \mathrm{M})($ Wako Pure Chemical Industries, Osaka, Japan) and 12 hours later, human/rat

6 ET-1 (10 nM) (Peptide Institute Inc., Osaka, Japan) was applied and incubated for 48

7 hours; the cell surface area was evaluated as describing below; and the sampling for

8 the $\left[{ }^{3} \mathrm{H}\right]$-leucine uptake, real-time PCR, and Pin1 expression was done in an individual

9 experiment. A Western blot was performed for ERK, JNK, and c-Jun, and the samples

10 were collected 30 minutes after the ET-1 stimulation. In the transient transfection

11 experiments, cardiomyocytes were transfected with an adenovirus for Pin1 or LacZ

12 (control) at a titer with 20 multiplicity of an infection (MOI); in the experiment of taking

13 photographs of cardiomyocytes, the adenovirus for EGFP (10 MOI) was transfected

14 additionally; 12 hours later after adenoviral transfection, cardiomyocytes were treated

15 with a vehicle or fluvastatin and harvested for 48 hours. There are some reasons why

16 we selected fluvastatin in this experiments; (1) as fluvastatin is known as one of

17 lipophilic statins contrasted to a hydrophilic statin, pravastatin, and is suspected to be

18 easily incorporated into the muscle cells, it suggested us that using fluvastatin leads the

19 easier evaluation for determining the inhibitory effect of statin on cardiomyocyte

20 hypertrophy; (2) other researchers previously reported that fluvastatin has the inhibitory

21 effect on cardimyocyte hypertrophy (Morikawa-Futamatsu et al., 2006); and (3) other

22 lipophilic statins, simvastatin and atorvastatin were reported to prevent cardiac

23 hypertrophy (Liu et al., 2008; Indolfi et al., 2002; Planavila et al., 2005).

24 Cardiomyocyte size measurement Cardiomyocytes were observed by microscope

25 (DM1L, Leica Japan, Tokyo, Japan) and captured by a charge-coupled device camera

26 (Olympus, Tokyo, Japan). The surface area of the attached cardiomyocytes on the dish 
1 (the area of cardiomyocytes in 2D image) for 10 cells/field with 5 fields in each group

2 was evaluated by the software NIH ImageJ ver 1.47 (National Institute of Health,

3 Bethesda, MD) and repeated by 3 times.

4 Protein synthetic rate The rates of protein synthesis in cultured neonatal rat cardiomyocytes were assessed by measuring the $\left[{ }^{3} \mathrm{H}\right]$-leucine uptake into acid-insoluble cellular materials as described previously (Sano et al., 2002; Shimojo et al., 2007). The cells were plated on 24 -well dishes at a density of $1 \times 10^{5}$ cells/well and pretreated with

8 fluvastatin or vehicle 12 hours before the ET-1 stimulation. Twenty-four hours after the

9 ET-1 treatment, $0.1 \mathrm{mCi} / \mathrm{ml}\left[{ }^{3} \mathrm{H}\right]$-leucine (GE Healthcare, Piscataway, $\mathrm{NJ}$ ) was added and the cells were incubated for 24 hours. The cells were finally fixed by $5 \%$ trichloroacetic acid and detached by $0.25 \%$ trypsin, and the cell residues were solubilized in $0.5 \mathrm{M} \mathrm{NaOH}$. Aliquots were counted with a scintillation counter (LS-6500 scintillation counter; Beckman Coulter, Fullerton, CA). In the transient transfection assay,

14 cardiomyocytes were transfected with an adenovirus for Pin1 or LacZ; 12 hours later,

15 fluvastatin or vehicle was added and cardiomyocytes were incubated for 48 hours;

$16\left[{ }^{3} \mathrm{H}\right]$-leucine was added and incubated for last 24 hours.

17 Gene expression analysis The total RNA from $1 \times 10^{6}$ cardiomyocytes was isolated using RNeasy (Qiagen, Valencia, CA) and was reverse transcribed to cDNA by a synthesis kit (Qiagen). The mRNA expression levels of the target genes were analyzed

20 by real-time quantitative PCR with a TaqMan probe using an ABI Prism 7700 sequence 21 detector (Applied Biosystems, Foster, CA), as described previously (Shimojo et al., 22 2007; Sakai et al., 2012). The commercially available gene-specific primers and 23 TaqMan probe sets were obtained from AppliedBiosystems. The PCR mixture (25 24 microL total volume) consisted of forward and reverse primers for each gene at $450 \mathrm{nM}$ each, FAM-labeled primer probes at $200 \mathrm{nM}$, and a TaqMan Universal PCR Master Mix

26 (AppliedBiosystems). PCR amplification was performed in duplicate as follows: 1 cycle 
1 at $95^{\circ} \mathrm{C}$ for $10 \mathrm{~min}$ and 40 cycles at $94^{\circ} \mathrm{C}$ for $15 \mathrm{~s}$ and $60^{\circ} \mathrm{C}$ for $1 \mathrm{~min}$. The quantitative

2 values of target mRNA were normalized by glyceraldehyde-3-phosphate

3 dehydrogenase (GAPDH) mRNA expression. The primers and probes sets were as

4 follows: atrial natriuretic peptide (ANP), Rn00561661_m1; B-type natriuretic peptide

5 (BNP), Rn00580641_m1; c-jun, Rn99999045_s1; and GAPDH, Rn01775763_g1.

6 Western blot Cardiomyocytes in a concentration of $2 \times 10^{6}$ from each group were

7 lysed on ice with a buffer ( $10 \mathrm{mM}$ Tris $\mathrm{HCl}, 150 \mathrm{mM} \mathrm{NaCl}, 1 \mathrm{mM}$ ethylenediamine- $\mathrm{N}, \mathrm{N}$,

$8 \quad \mathrm{~N}$ ', N'-tetraacetic acid [EDTA], $0.1 \%$ sodium dodecyl sulfate [SDS]), as described

9 previously (Shimojo et al. 2007; Sakai et al. 2012). The protein concentration of the

10 supernatant was determined with a bicinchoninic acid protein assay (Pierce, Rockford,

11 IL). Samples were run on an SDS-polyacrylamide gel electrophoresis (PAGE), using

$1210 \%$ or gradient $10-17.5 \%$ polyacrylamide gels, and electrotransferred to a PVDF

13 membrane. To reduce any nonspecific binding, the membrane was blocked for $1 \mathrm{~h}$ at

14 room temperature with $5 \%$ BSA or skim milk in tris-buffered saline (TBS) containing

$150.1 \%$ Tween 20 (TBST). Thereafter, the membrane was incubated overnight at $4^{\circ} \mathrm{C}$ with

16 primary antibodies. We incubated the membrane with a horseradish

17 peroxidase-conjugated goat anti-rabbit antibody or horse anti-mouse antibody (Cell

18 Signaling Technology, Boston, MA) at 1:10000 in a TBST at room temperature for 60

$19 \min$. The blots were visualized with an enhanced chemiluminescence detection system

20 (GE Healthcare), exposed to X-ray film or captured by CCD camera system (AE-6981,

21 Atto Corporation, Tokyo, Japan), and analyzed by CS Analyzer (Atto Corporation). The

22 following was used as the first antibodies: rabbit anti-Pin1, rabbit anti-phospho-Pin1,

23 rabbit anti-p44/42 MAP Kinase, rabbit anti-phospho-p44/42 MAP Kinase, rabbit

24 anti-SAPK/JNK, rabbit anti-phospho-SAPK/JNK, rabbit anti-C-Jun, rabbit

25 anti-phospho-c-Jun (these antibodies were purchased from Cell Signaling Technology),

26 mouse anti- $\beta$-actin (Sigma-Aldrich, St. Louis MO), and mouse anti-V5 (Life Technology). 
1 Statistical Analysis The values are shown as means \pm SE. Data were compared by a one-way ANOVA with a Turkey-Kramer's HSD using the JMP ver 8.0 statistical software

3 (SAS Institute, Cary, NC). Differences were considered significant at a $\mathrm{P}<0.05$.

\section{$5 \quad$ Results}

6 Cardiomyocyte hypertrophy and fluvastatin: The fluvastatin treatment markedly

7 attenuated the increase in cell surface area stimulated by ET-1 in a dose-dependent

8 manner (Figure 1A); the treatment with only fluvastatin alone slightly and significantly

9 decreased the cardiomyocyte surface area more than the dosage of $1 \mu \mathrm{M}$ (Figure $1 \mathrm{~A}$ ).

10 The fluvastatin treatment markedly inhibited the ET-1-induced increase of the protein

11 synthesis evaluated by $\left[{ }^{3} \mathrm{H}\right]$-leucine incorporation in a dose-dependent manner (Figure

12 1B); the treatment with fluvastatin alone significantly suppressed the incorporation only

13 at the dosage of $10 \mu \mathrm{M}$ (Actual values were followings: ET-1 [E] 0 [nM], Fluvastatin [F] 0

$14[\mu \mathrm{M}], 5125 \pm 294[\mathrm{cpm}$, mean \pm SE]; E0, F0.1, $4498 \pm$ 405; E0, F1, $3791 \pm 125 ; \mathrm{E} 0, \mathrm{~F} 10$

$152415 \pm 149 ; E 10, F 0,7348 \pm 406 ; E 10, F 0.1,7144 \pm 677 ; E 10, F 1,5159 \pm 29 ; E 10, F 10$

$163586 \pm 89)($ Figure 1B). These data indicated that fluvastatin has an inhibitory effect on

17 cardiomyocyte hypertrophy provoked by ET-1. Moreover, there was a possibility that

18 fluvastatin has a cytotoxic effect at a dosage of $10 \mu \mathrm{M}$, because of the marked

19 suppression in protein synthesis at this dosage and of the report that $3 \mu \mathrm{M}$ fluvastatin

20 induced apoptosis in cardiomyocyte (Ogata et al., 2002); therefore, we used fluvastatin

21 at a dosage of $1 \mu \mathrm{M}$ when we analyzed the effect of fluvastatin on cardiomyocyte. The

22 mRNA expression of the cardiomyocyte hypertrophy markers ANP and BNP was

23 significantly increased by ET-1, and fluvastatin significantly decreased both expressions 
1 (Figures 2A, 2B), suggesting that the inhibitory effect of fluvastatin on hypertrophy was

2 also confirmed by the alteration of the hypertrophy markers.

3 Intracellular signaling of cardiomyocyte hypertrophy and fluvastatin: Fluvastatin

4 markedly decreased both phospho-JNK and phospho-c-Jun level activated by ET-1

5 (Figure 3B), suggesting the inhibition of the JNK pathway by fluvastatin; however,

6 fluvastatin did not alter the phospho-ERK level augmented by ET-1 (Figure 3A). These

7 data suggest that the inhibitory effect of fluvastatin on cardiomyocyte hypertrophy is

8 mediated mainly via the JNK pathway rather than ERK. One of the downstream targets

9 of JNK is c-Jun, which consists the transcription factor activator protein-1 (AP-1) as the

10 homodimer by itself or heterodimer with c-Fos. The increased expression of c-jun

11 mRNA induced by ET-1 was significantly suppressed by fluvastatin (Figure 4A);

12 phospho-c-Jun was augmented by ET-1, and fluvastatin markedly attenuated the

13 phosphorylation (Figure 4B). These data suggest that the inhibitory effect of fluvastatin

14 on cardiomyocyte hypertrophy is predominantly associated with the JNK to c-Jun

15 signaling, although ET-1 mobilizes both the ERK and JNK pathways.

16 Involvement of Pin1 in cardiomyocyte hypertrophy. The phospho-Pin1 level was

17 significantly lower in the cardiomyocytes stimulated by ET-1 than the control (without

18 ET-1); the reduced phospho-Pin1 level was reversed to the control level by fluvastatin

19 treatment (Figure 5). The data suggest that a functional alteration of Pin1 occurred in

20 the hypertrophied cardiomyocyte induced by ET-1, and that fluvastatin inhibited the

21 ET-1-induced cardiomyocyte hypertrophy partly via the normalization of Pin1

22 phosphorylation. To study the role of Pin1 in the cardiomyocyte hypertrophy and how

23 fluvastatin is involved in the Pin1 regulation, transient transfection of Pin1 was done

24 using an adenovirus vector (Figures 6A-6E). Morphologically, Pin1 overexpression

25 induced cardiomyocyte hypertrophy (Figures 6A, 6B, 6D) and fluvastatin inhibited the

26 Pin1-induced hypertrophy (Figures 6B, 6C, 6D). These data suggested that a gain of 
1 the Pin1 function induces cardiomyocyte hypertrophy and that Pin1-induced

2 hypertrophy is suppressed by fluvastatin. In Western blot analysis, the V5-tagged Pin1

3 (Pin1-V5) transgene product was verified by the anti-V5 antibody; the expression level

4 of endogenous Pin1 did not differ among 3 groups (LacZ, Pin1 without fluvastatin, and

$5 \quad$ Pin1 with fluvastatin); exogenously transfected Pin1-V5 was detected at the upper part

6 compared to endogenous Pin1 because of the increase in molecular weight;

7 phosphorylated Pin1 level was markedly increased in Pin1-V5 transfected groups

8 compared to LacZ group in this experimental condition and phosphorylated Pin1 level

9 was tended to be increased in fluvastatin-treated Pin1 group compared to

10 vehicle-treated Pin1 group (Figure 6E).

11

12

\section{Discussion}

14 This is the first report that ET-1-induced cardiomyocyte hypertrophy is mediated

15 through the Pin1 activation and that the inhibitory effect of fluvastatin on cardiomyocyte

16 hypertrophy would partly be attributed to the suppression of the Pin1 function. These

17 findings suggest that the ET-1-induced cardiomyocyte hypertrophy is mediated through

18 the activation of Pin1, which may be a key molecule determining the size of

19 hypertrophied cardiomyocytes; furthermore, this is the first study to demonstrate that

20 the inhibitory effect of fluvastatin on ET-1-induced hypertrophy would partly be attributed

21 to the suppression of the Pin1 function by affecting the signaling from JNK to c-Jun.

\section{Pin1 involvement in the cardiomyocyte hypertrophy}

24 Pin1 is a small protein with an $\mathrm{N}$-terminal WW domain and a C-terminal PPlase

25 domain (Lu et al., 2007). The WW domain binds phosphorylated Ser/Thr-Pro

26 (pSer/Thr-Pro) motifs; the PPlase domain catalyzes cis/trans-isomerization of the 
1 proline-containing peptides; thus, Pin1 changes the activity of interacting proteins (Lu et

2 al., 2007). It has been reported that Pin1 is overexpressed in breast cancer and

3 promotes Ras signaling by increasing the transcriptional activity of c-Jun (Wulf et al.,

4 2001). In the present study, ET-1 activated the ERK, JNK, and c-Jun, a downstream

5 target of JNK, simultaneously, and ET-1 attenuated the phosphorylated Pin1, an inactive

6 form of Pin1, suggesting that ET-1 increases the catalytic activity of Pin1. Human c-Jun

7 has a phosphorylated Ser63/Pro73 sequence and is known to be one of the Pin1 target

8 proteins (Wulf et al., 2001). Therefore, Pin1 may participate in ET-1-induced

9 cardiomyocyte hypertrophy partly via the activation of c-Jun, a component of the

10 transcription factor activator protein $(\mathrm{AP})-1$.

11 Recently, it has been reported that human JNK1, an upstream precursor of c-Jun, 12 also has four pSer/Thr-Pro motifs and Thr-183 on JNK1 is critical for JNK1 activation 13 and Pin1 binding (Park et al., 2012); therefore, Pin1 might participate in ET-1-induced 14 hypertrophy via the activation of JNK as well as c-Jun. Furthermore, it has also been 15 reported that ERK and c-Fos, a downstream target of ERK, have pSer/Thr-Pro motifs 16 and that Pin1 can bind to ERK and c-Fos (Monje et al., 2005; Toko et al., 2013); it 17 suggests that Pin1 is involved in cardiomyocyte hypertrophy by cooperating with c-Jun 18 and c-Fos to regulate the AP-1-dependent gene transcription upon activation of the 19 MEK family members. In addition, the C-terminal domain of the largest subunit in RNA 20 polymerase II, the global regulator machinery of transcription, is phosphorylated at 21 Ser-2 and Ser-5 residues prior to the Pro at hypertrophy (Sano et al., 2004; Sakai et al., 22 2012); Pin1 would contribute to the activation of RNA polymerase II and be involved in 23 the hypertrophic responses. Therefore, Pin1 may play an important role in the 24 development of cardiomyocyte hypertrophy not only via the activation of c-Jun but also 25 via several kinds of functional molecules. 


\section{Inhibitory effect of fluvastatin on ET-1-induced cardiomyocyte hypertrophy}

Some researchers have reported the inhibitory effects of statins on cardiac

3 hypertrophy both in vivo and in vitro; simvastatin prevented cardiac hypertrophy induced

4 by a pressure overload through p21ras inactivation (Indolfi et al., 2002) and angiotensin

$5 \quad$ Il-induced hypertrophy through an antioxidant mechanism involving Rac1 inhibition

6 (Takemoto et al., 2001); and fluvastatin inhibited cardiac hypertrophy via the suppression of cyclin D1 linked to Rho kinase (Morikawa-Futamatsu et al., 2006). The

8 finding of the present study that fluvastatin inhibited the cardiomyocyte hypertrophy

9 induced by ET-1 is also expected from the previous research mentioned above, and not a surprising idea because cardiomyocyte stimuli including angiotensin II, ET-1, and a

11 pressure overload finally activate similar signaling pathways, i.e., the activation of the MEK family and transcription factor AP-1 (Heineke and Molkentine. 2006).

The new finding of the present study was that the inhibitory effect of fluvastatin on

14 hypertrophy could partly be mediated via the suppression of the Pin1 function; one of

15 the Pin1 targets inactivated by fluvastatin was suspected to be the JNK to c-Jun

16 pathway rather than ERK, because fluvastatin predominantly decreased the expression

17 of phospho-JNK and phospho-c-Jun, but not phospho-ERK, under ET-1 stimulation.

18 Cyclin D1, whose function is suppressed by statins, is also known to be a target of Pin1

19 (Liou et al., 2002); therefore, there is a possibility that the involvement of cyclin D1

20 inhibition by fluvastatin on the anti-hypertrophy effect is partly mediated via the 21 suppression of Pin1.

22 In the transient transfection assay using Pin1 adenovirus vector, the gain of the Pin1

23 function by the Pin1 overexpression caused cardiomyocyte hypertrophy, whereas

24 fluvastatin attenuated the Pin1-induced hypertrophy. The ET-1-induced reduction in 25 phospho-Pin1 level was reversed by fluvastatin (Fig 5), whereas the expression level of 26 phospho-Pin1 did not differ significantly between in fluvastatin treated Pin1 group and in 
1 vehicle-treated Pin1 group (Figure 6E). Therefore, we consider a mechanism for an

2 inhibition of Pin1-induced cardiomyocyte hypertrophy by fluvastatin with no alteration of

3 the phospho-Pin1 level as followings. Because it has been reported that

4 death-associated protein kinase 1 (DAPK1) inhibits the catalytic activity of Pin1 (Lee et

5 al., 2011), it would be suspected that the suppressive effect of statins on Pin1-mediated

6 hypertrophic response is partly be attributed to the magnitude of DAPK1 activation.

7 Alternatively, the following discussion may be also possible. The overexpression

8 experiment of Pin1 transgene, which was forcedly expressed by CMV promoter and out

9 of the transcriptional control by primarily or secondarily statin-regulated transcriptional

10 factors, is an artificial condition. Thus, the transgene product may be a large amount

11 compared with the endogenous Pin1 protein; therefore, we could not detect a significant

12 difference of phospho-Pin1 expression level between in vehicle-treated Pin1 group and

13 in fluvastatin-treated Pin1. In addition to above consideration, the precise mechanism

14 for statins in the inactivation of Pin1 function still remains to be resolved.

\section{Conclusion}

17 The present study revealed for the first time that (1) ET-1-induced cardiomyocyte 18 hypertrophy is mediated through the activation of Pin1, which may be a key molecule 19 determining the size of hypertrophied cardiomyocyte; (2) the inhibitory effect of

20 fluvastatin on ET-1-induced hypertrophy would partly be attributed to the suppression of

21 the Pin1 function; (3) the pathway from JNK to c-Jun would be a major target of Pin1.

22 These findings firstly suggest that Pin1 is an important molecule for aggravating cardiac

23 hypertrophy and that the inhibition of the Pin 1 function by HMG-CoA reductase

24 inhibitors is a novel mechanism for pleiotropic effects of them.

26 Acknowledgements 
1 This work was supported by the Ministry of Education, Science, Sports, and Culture

2 of Japan Grants-in-Aid for Scientific Research (25293125, 23592025, 25861714,

$325860581,22790687,25670757,23500835,22390334,23659279,24590654)$ and a

4 grant from the Miyauchi Project of Tsukuba Advanced Research Alliance at the

5 University of Tsukuba. We thank Sayaka Inoue, Ayami Nakamura, Naomi Koharazawa,

6 and Mari Nakagawa for their technical assistance.

7

\section{Conflict of interest}

$9 \quad$ There are no conflicts of interests. 


\section{$1 \quad$ References}

- Davenport AP. International union of pharmacology. XXIX. Update on endothelin

3 receptor nomenclature. Pharmacol Rev. 2002; 54: 219-26.

- Heineke J, Molkentin JD. Regulation of cardiac hypertrophy by intracellular

$5 \quad$ signaling pathways. Nat Rev Mol Cell Biol. 2006; 7: 589-600.

7 regulatory mechanisms. J Pharmacol Sci. 2013; 123: 85-101. cardiac hypertrophy induced by pressure overload and inhibits p21ras activation. Circulation. 2002; 106: 2118-2124. hypertrophy is inhibited by activation of peroxisome proliferator-activated receptor-alpha partly via blockade of c-Jun $\mathrm{NH} 2$-terminal kinase pathway. 
- Ito H, Hirata Y, Hiroe M, Tsujino M, Adachi S, Takamoto T, Nitta M, Taniguchi K,

2 Marumo F. Endothelin-1 induces hypertrophy with enhanced expression of

3 muscle-specific genes in cultured neonatal rat cardiomyocytes. Circ Res. 1991; 69:

$4209-215$.

- Ito H. Endothelins and cardiac hypertrophy. Life Sci. 1997; 61: 585-593.

7 disease and myocardial infarction. Cardiol Rev. 2013;21:249-56.

- Lee TH, Pastorino L, Lu KP. Peptidyl-prolyl cis-trans isomerase Pin1 in ageing,

$9 \quad$ cancer and Alzheimer disease. Expert Rev Mol Med. 2011; 13: e21.

10 - Lee TH, Chen CH, Suizu F, Huang P, Schiene-Fischer C, Daum S, Zhang YJ,

11 Goate A, Chen RH, Zhou XZ, Lu KP. Death-associated protein kinase 1

12 phosphorylates Pin1 and inhibits its prolyl isomerase activity and cellular function.

13 Mol Cell. 2011; 42: 147-159. 
- Liu J, Shen Q, Wu Y. Simvastatin prevents cardiac hypertrophy in vitro and in

2 vivo via JAK/STAT pathway. Life Sci. 2008; 82: 991-996.

- Lu KP, Hanes SD, Hunter T. A human peptidyl-prolyl isomerase essential for

4 regulation of mitosis. Nature. 1996; 380: 544-547.

- Lu KP and Zhou XZ. The prolyl isomerase PIN1: a pivotal new twist in

6 phosphorylation signaling and disease. Nat Rev Mol Cell Biol. 2007; 3:

$7 \quad 619-629$.

8 - Miyauchi T and Goto K. Endothelins. (Edited by Abba J. Kastin) "Handbook of

9 Biologically Active Peptides (Second Edition)", Academic Press (Elsevier Inc.),

$10 \quad$ Chapter 190, pp $1402-1407,2013$.

11 - Monje P, Hernandez-Losa J, Lyons RJ, Castellone MD, Gutkind JS. Regulation

12 of the transcriptional activity of c-Fos by ERK: A novel role for the prolyl isomerase

13 Pin1. J Biol Chem. 2005; 280: 35081-35084.

- Morikawa-Futamatsu K, Adachi S, Maejima Y, Tamamori-Adachi M, Suzuki J, 
$1 \quad$ D1. Life Sci. 2006; 79: 1380-1390.

2

- Ogata Y, Takahashi M, Takeuchi K, Ueno S, Mano H, Ookawara S, Kobayashi E,

$3 \quad$ Ikeda $\mathrm{U}$, Shimada K. Fluvastatin induces apoptosis in rat neonatal cardiac

4 myocytes: A possible mechanism of statin-attenuated cardiac hypertrophy. $\mathrm{J}$

$5 \quad$ Cardiovasc Pharmacol. 2002; 40: 907-915.

6 - Park JE, Lee JA, Park SG, Lee DH, Kim SJ, Kim H, Uchida C, Uchida T, Park BC,

7 Cho S. A critical step for JNK activation: isomerization by the prolyl isomerase

$8 \quad$ Pin1. Cell Death Differ. 2012; 19: 153-161.

9 - Planavila A, Laguna J, Vazquez-Carrera M. Atrovastatin improves peroxisome

10 proliferator-activated receptor signaling in cardiac hypertrophy by preventing

11 nuclear factor-kappa B activation. Biochem Biophysic Acta. 2005; 1687: 76-83.

12 - Sakai S, Miyauchi T, Sakurai T, Kasuya Y, Ihara M, Yamaguchi I, Goto K,

13 Sugishita Y. Endogenous endothelin-1 participates in the maintenance of cardiac

14 function in rats with congestive heart failure. Marked increase in endothelin-1

15 production in the failing heart. Circulation. 1996a; 93: 1214-1222. 
1 Inhibition of myocardial endothelin pathway improves long-term survival in heart

2 failure. Nature 1996b; 384: 353-355,.

- Sakai S, Miyauchi T, Yamaguchi I. Long-term endothelin receptor antagonist

4 administration improves alterations in expression of various cardiac genes in failing

$5 \quad$ myocardium of rats with heart failure. Circulation. 2000; 101: 2849-2853.

- Sakai S, Kimura T, Wang Z, Shimojo N, Maruyama H, Homma S, Kuga K,

7 Yamaguchi I, Aonuma K, Miyauchi T. Endothelin-1-induced cardiomyocyte

8 hypertrophy is partly regulated by transcription factor II-F interacting C-terminal

9 domain phosphatase of RNA polymerase II. Life Sci. 2012; 91: 572-577.

10 - Sano M, Wang SC, Shirai M, Scaglia F, Xie M, Sakai S, Tanaka T, Kulkarni PA,

11 Barger PM, Youker KA, Taffet GE, Hamamori Y, Michael LH, Craigen WJ,

12 Schneider MD. Activation of cardiac Cdk9 represses PGC-1 and confers a

13 predisposition to heart failure. EMBO J 2004; 23: 3559 - 3569. 
1 hypertrophy in vitro through the suppression of TGF-beta1 and phosphorylated

2 JNK. Am J Physiol Heart Circ Physiol. 2006; 291: H835-845.

3 - Shimojo N, Jesmin S, Zaedi S, Otsuki T, Maeda S, Yamaguchi N, Aonuma K,

4 Hattori Y, and Miyauchi T. Contributory role of VEGF overexpression in

5 endothelin-1-induced cardiomyocyte hypertrophy. Am J Physiol- Heart \& Circ

$6 \quad$ Physiol 2007; 293: H474-H481.

7 - Tajiri K, Shimojo N, Sakai S, Machino-Ohtsuka T, Imanaka-Yoshida K, Hiroe M,

8 Tsujimura Y, Kimura T, Sato A, Yasutomi Y, Aonuma K. Pitavastatin regulates

9 helper T-cell differentiation and ameliorates autoimmune myocarditis in mice.

$10 \quad$ Cardiovasc Drugs Ther. 2013; 27: 413-427.

11 - Takemoto M, Node K, Nakagami H, Liao Y, Grimm M, Takemoto Y, Kitakaze M,

12 Liao JK. Statins as antioxidant therapy for preventing cardiac myocyte

13 hypertrophy. J Clin Invest. 2001; 108: 1429-1437.

14 - Toko H, Konstandin MH, Doroudgar S, Ormachea L, Joyo E, Joyo AY, Din S, Gude

15 NA, CollinsB, Volkers M, Thuerauf DJ, Glembotski CC, Chen C, Lu KP, Muller OJ, 
1 Uchida T, Sussman MA. Regulation of cardiac hypertrophic signaling by prolyl

$2 \quad$ isomerase Pin1. Circ Res. 2013; 112: 1244-1252.

3

- Yanagisawa M, Kurihara H, Kimura S, Tomobe Y, Kobayashi M, Mitsui Y, Yazaki Y,

4 Goto K, Masaki T. A novel potent vasoconstrictor peptide produced by vascular

$5 \quad$ endothelial cells. Nature 1988; 332: 411-415.

6 - Yorikane R, Sakai S, Miyauchi T, Sakurai T, Sugishita Y, Goto K. Increased

7 production of endothelin-1 in the hypertrophied rat heart due to pressure overload.

$8 \quad$ FEBS Lett 1993; 332: 31-34.

9 - Yue TL, Gu JL, Wang C, Reith AD, Lee JC, Mirabile RC, Kreutz R, Wang Y,

10 Maleeff B, Parsons AA, Ohlstein EH. Extracellular signal-regulated kinase plays

11 an essential role in hypertrophic agonists, endothelin-1 and phenylephrine-induced

12 cardiomyocyte hypertrophy. J Biol Chem. 2000; 275: 37895-37901.

13 - Wierzbicki AS, Poston R, Rerro A. The lipid and non-lipid effects of statins.

$14 \quad$ Pharmacol Ther. 2003; 99: 95-112. 
1 the transcriptional activity of c-Jun towards cyclin D1. EMBO J 2001; 20:

2

3459-3472.

3 


\section{$1 \quad$ Figure Legends}

2 Figure 1. Fluvastatin inhibited ET-1-induced cardiomyocyte hypertrophy. ET-1 (10

$3 \mathrm{nM}$ )-induced cardiomyocyte hypertrophy was inhibited by fluvastatin in a dose

4 dependent manner evaluated by the cell surface area (10 cells/field with 5 fields

$5 \quad[n=50]$ in each combination) $(A)$ and by $\left[{ }^{3} \mathrm{H}\right]$-leucine uptake (4 wells $[n=4]$ in each

6 combination) (B). Data were analyzed by a one-way ANOVA and expressed as the

7 mean \pm SE. ${ }^{*} \mathrm{P}<0.05,{ }^{* *} \mathrm{P}<0.01$ compared with the control (treatments without $\mathrm{ET}-1$ and

8 fluvastatin). ${ }^{\#} \mathrm{P}<0.05,{ }^{\# \#} \mathrm{P}<0.01$ compared with only $\mathrm{ET}-1$ treatment.

9

10 Figure 2. Effects of fluvastatin (Flu, $1 \mu \mathrm{M})$ and ET-1 (10 nM) treatments on the 11 expression of the cardiomyocyte hypertrophy markers. ANP mRNA ( $n=6$ for each group)

12 (A) and BNP mRNA ( $\mathrm{n}=6$ for each group) (B) evaluated by RT-PCR. GAPDH mRNA 13 was used as an internal control. Data were analyzed by a one-way ANOVA and 14 expressed as the mean $\pm \mathrm{SE}$. ${ }^{*} \mathrm{P}<0.05$ compared with the control (treatments without 15 ET-1 and fluvastatin). ${ }^{\#} \mathrm{P}<0.05$ compared with only ET-1 treatment.

17 Figure 3. Effects of fluvastatin (Flu, $1 \mu \mathrm{M})$ and ET-1 $(10 \mathrm{nM})$ treatments on the expression of ERK and JNK evaluated by a Western blot. A, Representative blots

19 showed the phospho-ERK and total ERK levels, and the bar graph represented the ratio 20 of the blot intensity of the phospho-ERK to the total ERK ( $n=4$ for each group); $\mathbf{B}$, blots 21 showed the phosph-JNK and total JNK, and the bar graph represented that of the 22 phospho-JNK to the total JNK ( $\mathrm{n}=4$ for each group). Data were expressed as the mean $23 \pm$ SE. ${ }^{* *} \mathrm{P}<0.01$ compared with the control (treatments without ET-1 and fluvastatin). $24 \quad$ \#\# $\mathrm{P}<0.01$ compared with ET-1 treatment. 
1 Figure 4. Effects of fluvastatin (Flu, $1 \mu \mathrm{M})$ and ET-1 $(10 \mathrm{nM})$ treatments on the

2 expression of c-Jun. A, Expression of c-jun mRNA expression, and the bar graph

3 represented the ratio of the $c$-jun expression to the GAPDH expression ( $n=6$ for each

4 group); B, representative Western blots showing the phospho-C-Jun and total C-Jun,

5 and the bar graph represented the ratio of the blot intensity of the phospho-c-Jun to the

6 total $c$-Jun ( $n=4$ for each group). Data were expressed as the mean $\pm S E .{ }^{* *} P<0.01$

7 compared with the control (treatments without ET-1 and fluvastatin). ${ }^{\#} \mathrm{P}<0.05$ compared

8 with ET-1 treatment.

9

10 Figure 5. Effects of fluvastatin $(\mathrm{Flu}, 1 \mu \mathrm{M})$ and ET-1 $(10 \mathrm{nM})$ treatments on the 11 expression of phospho-Pin1 evaluated by a Western blot. Representative blots showing 12 the phospho-Pin1 and total Pin1 levels, and the bar graph represented the ratio in the 13 blot intensity of the phospho-Pin1 to the total Pin1. Relative expression level of the 14 phospho-Pin1 to the total Pin1 ( $n=4$ for each group). Data were expressed as the mean $15 \pm \mathrm{SE}$. ${ }^{* *} \mathrm{P}<0.01$ compared with the control (no treatments). ${ }^{\# \#} \mathrm{P}<0.01$ compared with 16 ET-1 treatment.

18 Figure 6. Cardiomyocyte hypertrophy induced by Pin1 overexpression was inhibited 19 by fluvastatin $(1 \mu \mathrm{M})$. Representative photographs of cardiomyocytes transiently 20 transfected by adenoviruses (20 MOI); (A) LacZ (control), (B) Pin1, (C) Pin1 treated 21 with fluvastatin. Cardiomyocytes were labeled by adenovirus for EGFP (10 MOI), 22 additionally. Bar represents $100 \mu \mathrm{M}$. D, The bar graph represented the $\left[{ }^{3} \mathrm{H}\right]$-leucine 23 uptake (4 wells [ $n=4$ ] in each combination). E, Representative Western blot showing the 24 phospho-Pin1, total Pin1, and V5 protein level overexpressed by the V5-tagged Pin1 adenovirus transfection. Data were expressed as the mean $\pm S E .{ }^{*} P<0.05,{ }^{* \star} P<0.01$ compared with LacZ transfection without fluvastatin treatment. ${ }^{\#} \mathrm{P}<0.05$ compared with 
1 Pin1 transfection without fluvastatin treatment. 
Figures $1 \mathrm{~A}$ and $1 \mathrm{~B} \quad$ Sakai S, et al.

A

(\%)

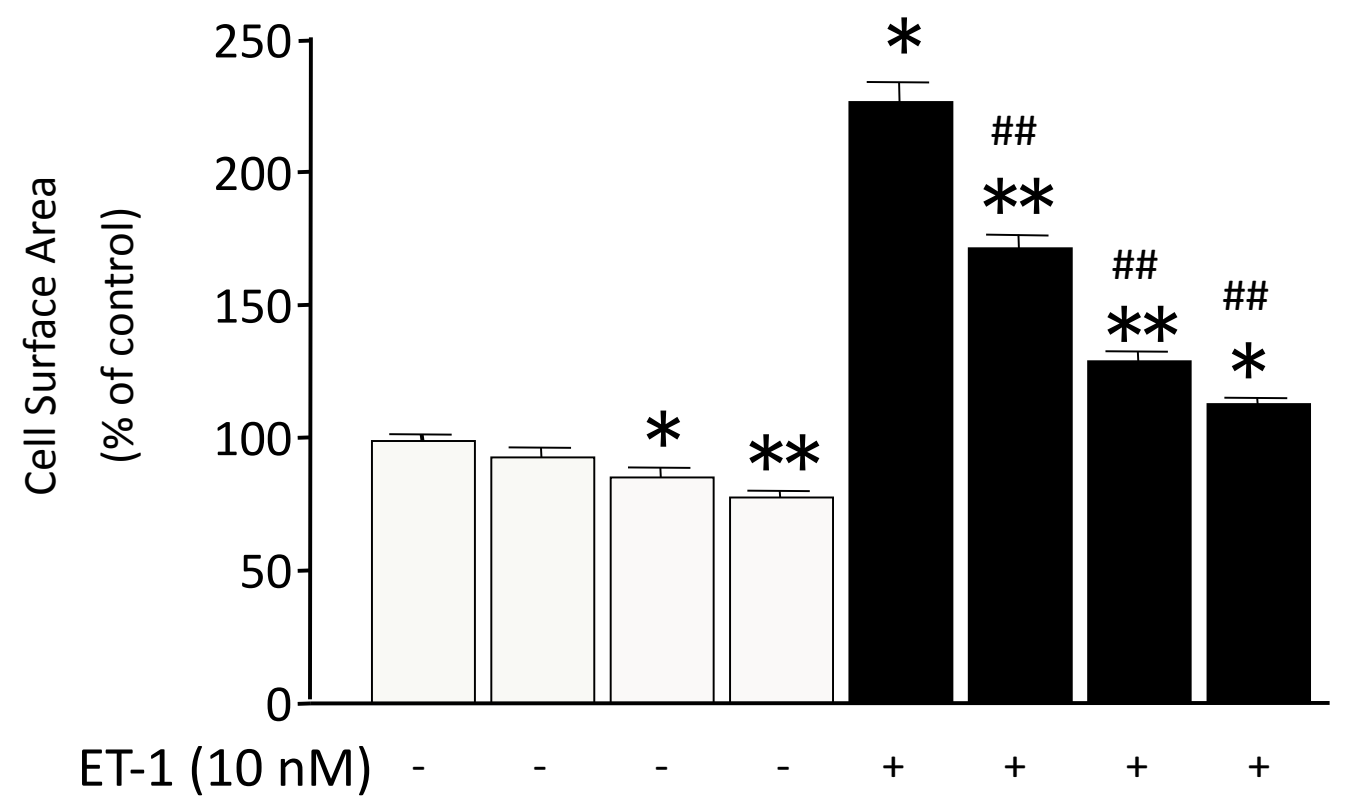

$\begin{array}{lllllllll}\text { Fluvastatin }(\mu \mathrm{M}) & - & 0.1 & 1 & 10 & - & 0.1 & 1 & 10\end{array}$

B

(\%)

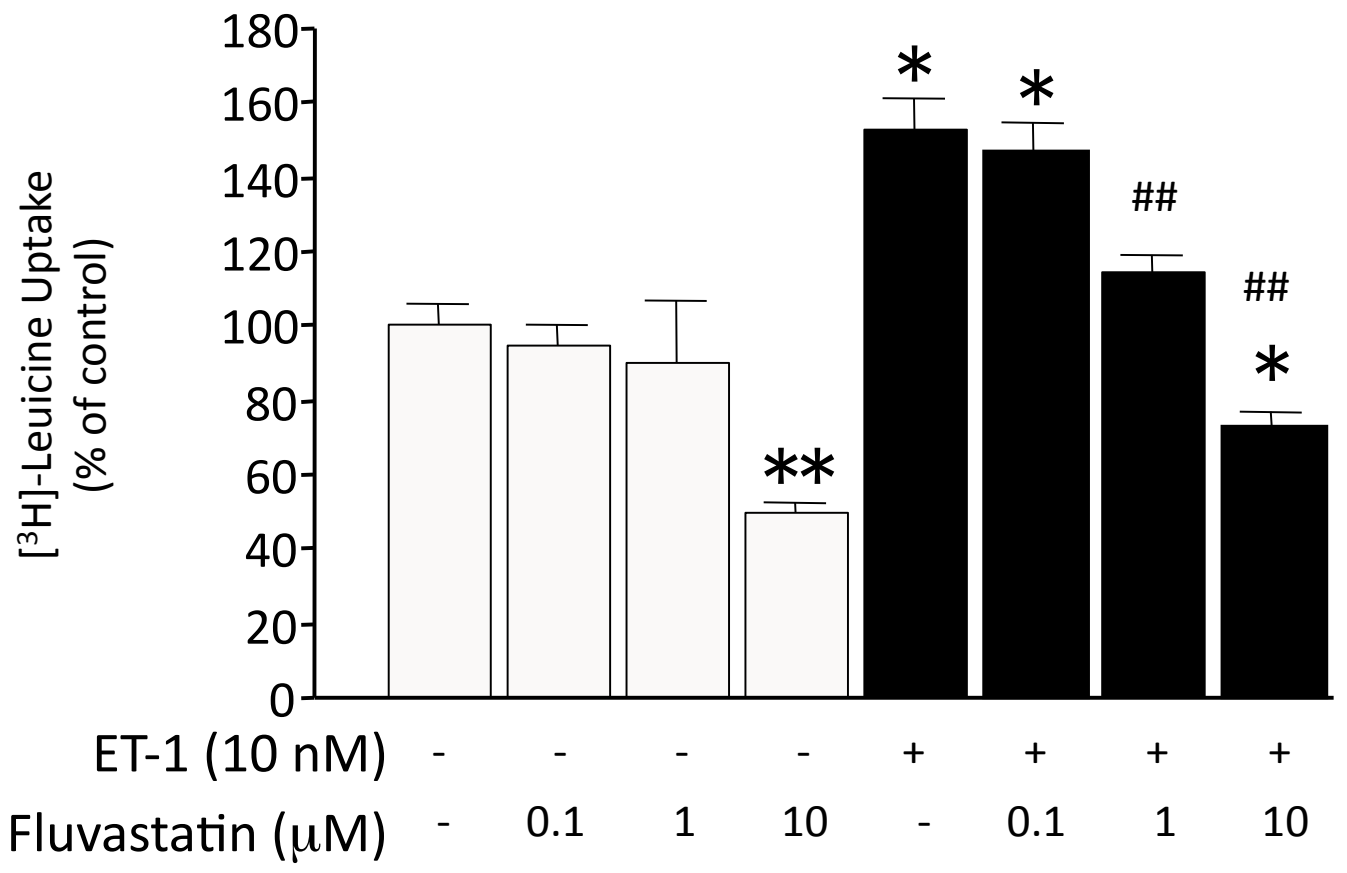


Figures 2A and 2B Sakai S, et al.

A

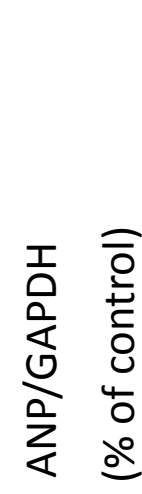

B

(\%)

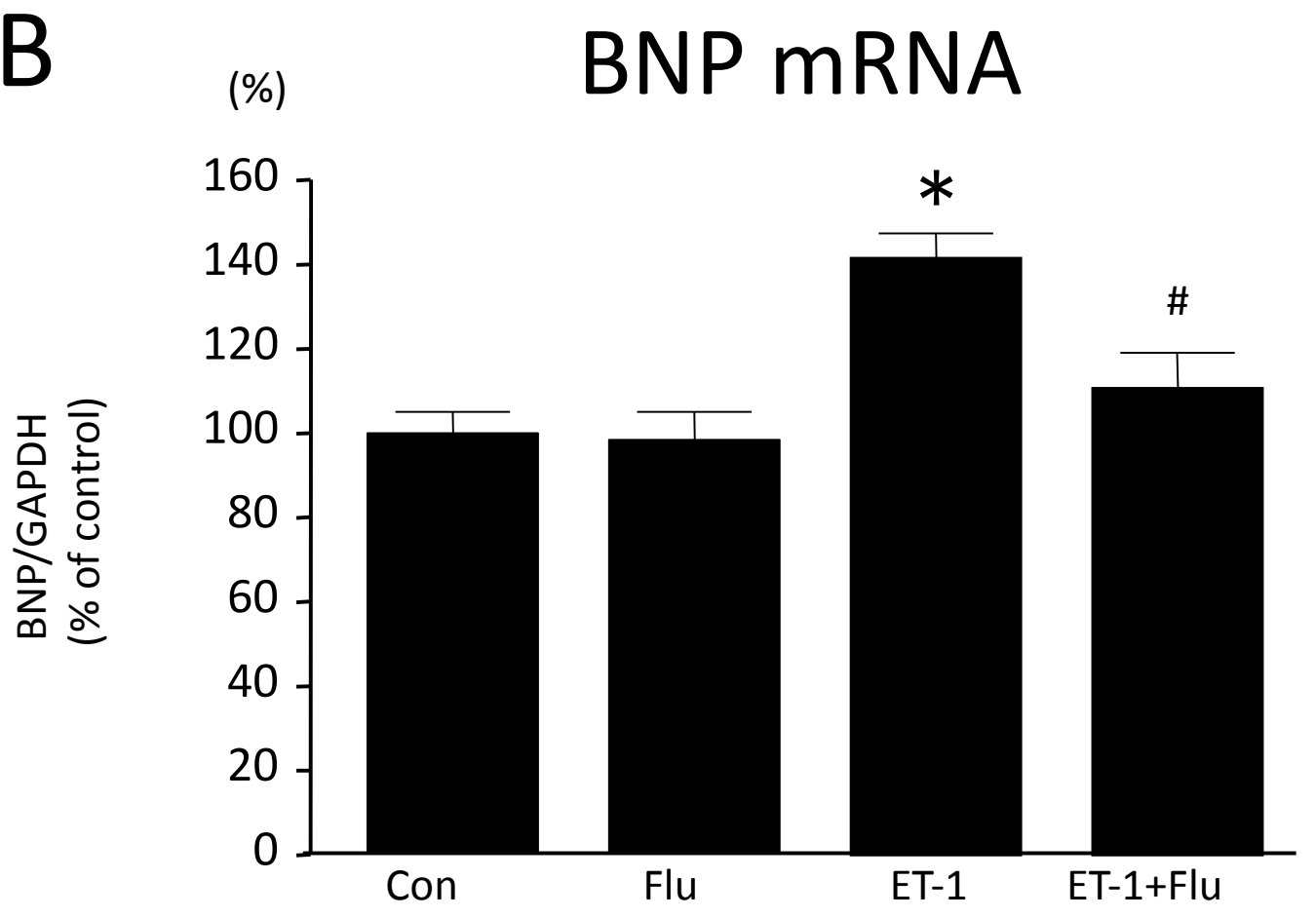

ANP mRNA

180

160

$80-$

$40-$ $20-$

Con

Flu

BNP MRNA

ET-1

ET-1+Flu

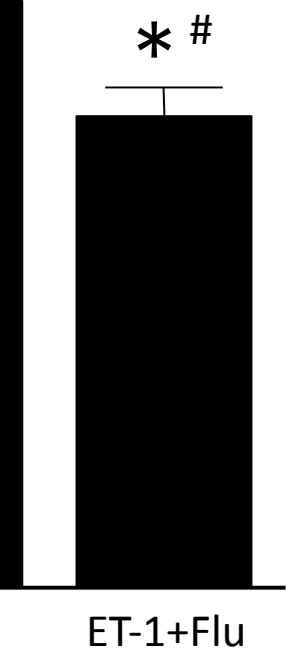

B 
Figures $3 A$ and $3 B$ Sakai $S$, et al.

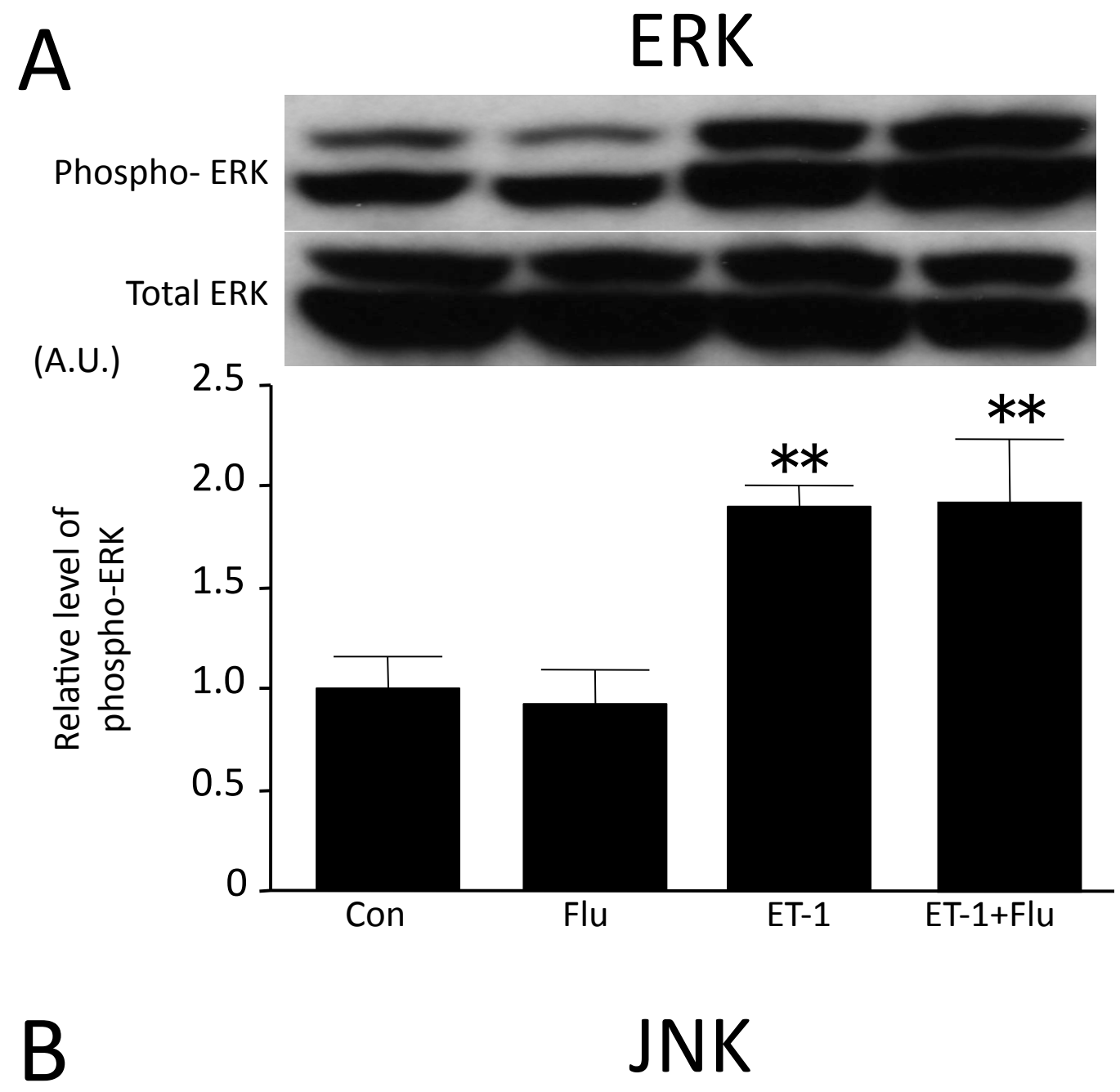

Phospho- JNK

Total JNK

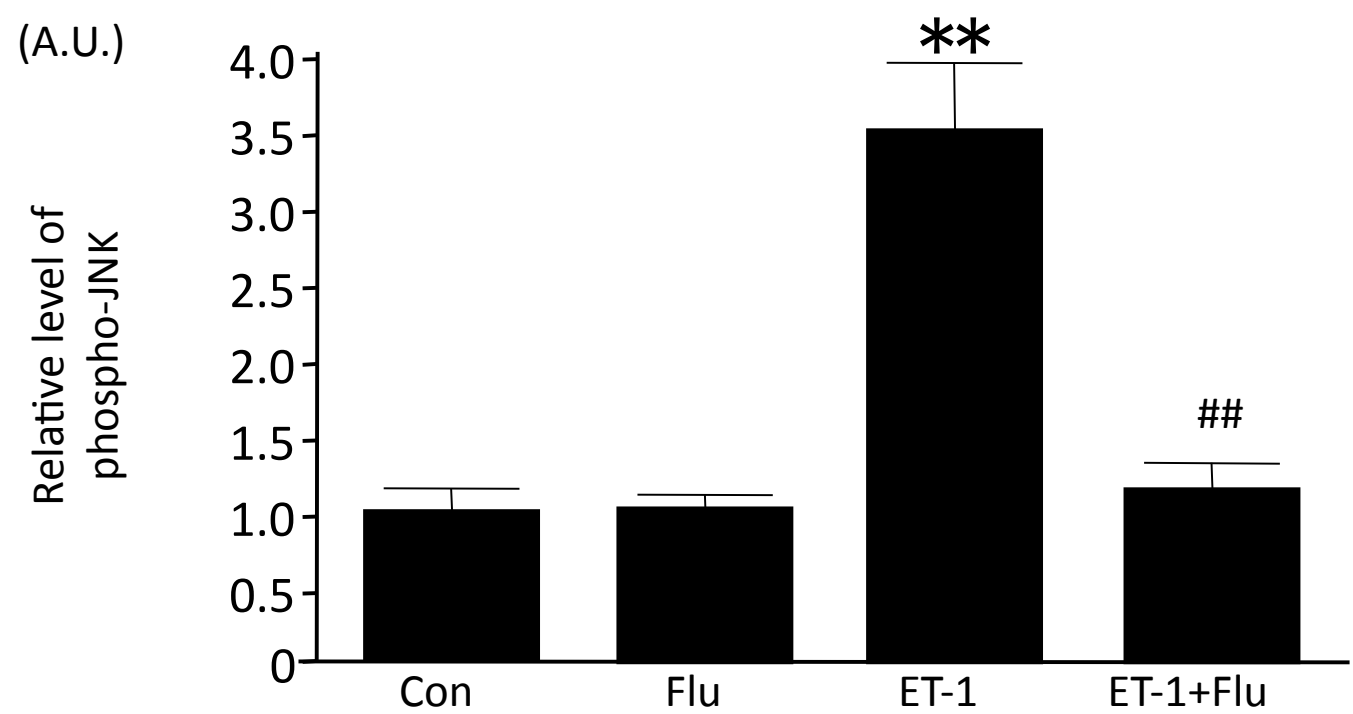


Figures $4 \mathrm{~A}$ and $4 \mathrm{~B}$ Sakai S, et al.

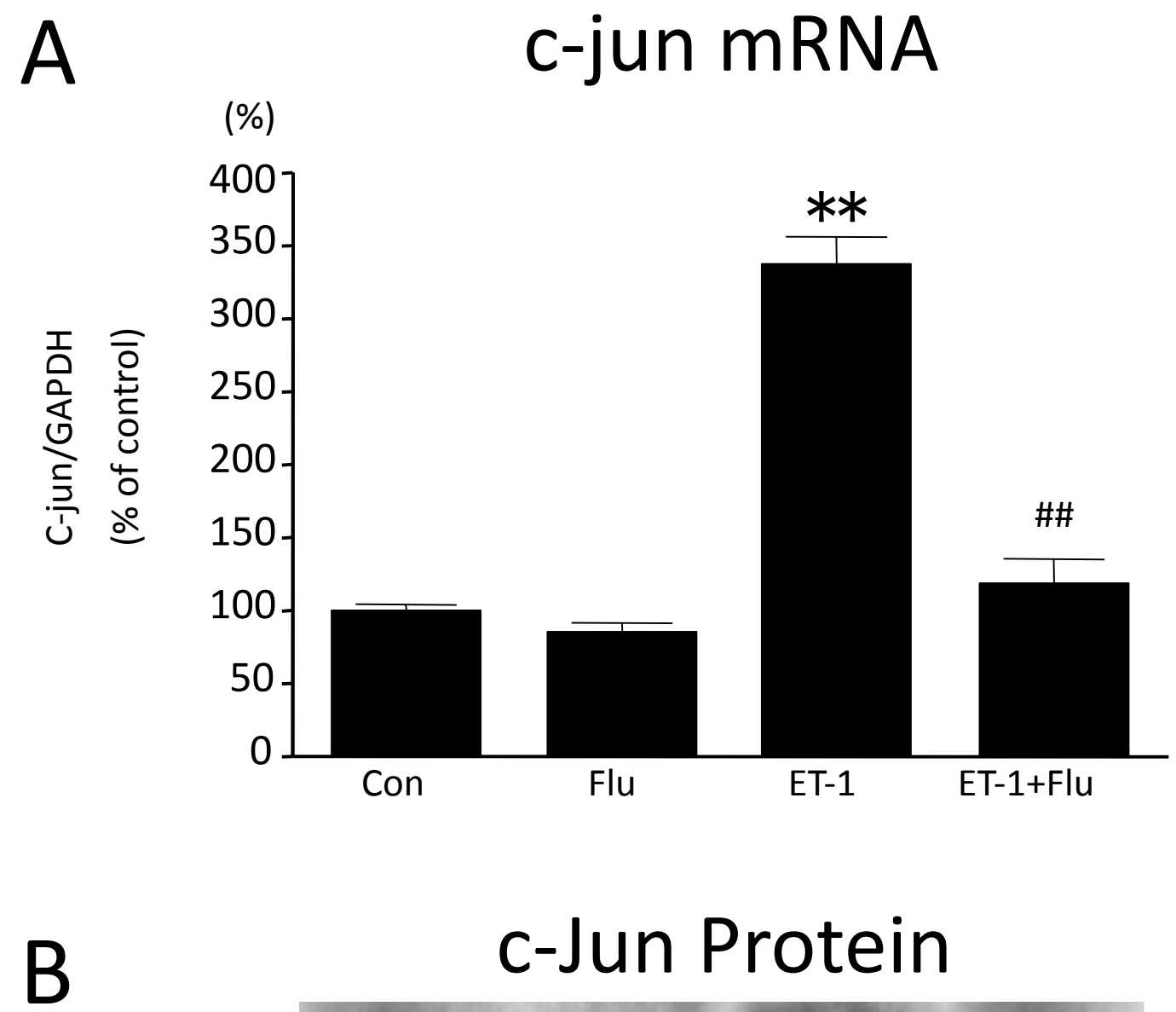

Phospho- c-Jun

Total c-Jun

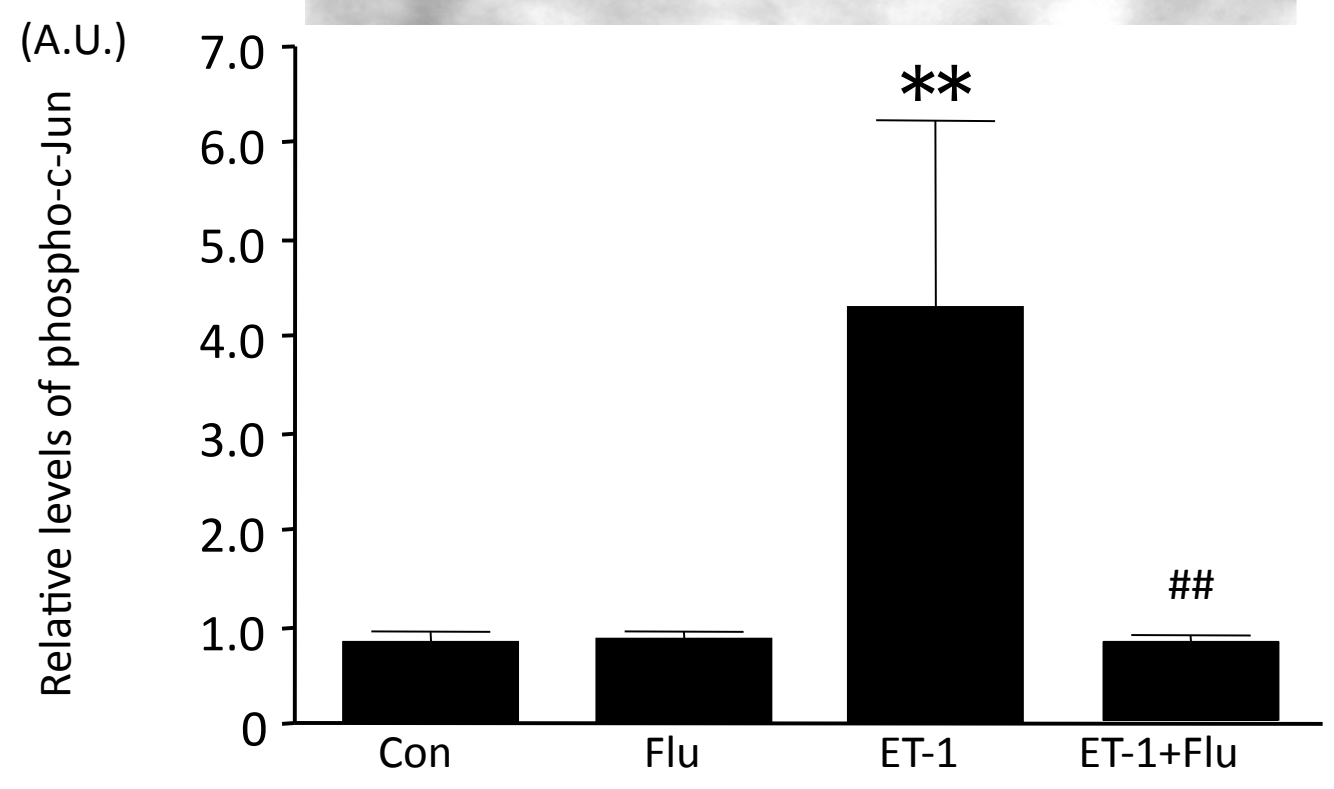


Figure 5

Figure 5 Sakai S, et al.

\section{Pin1}

Phospho- Pin1

Total Pin1

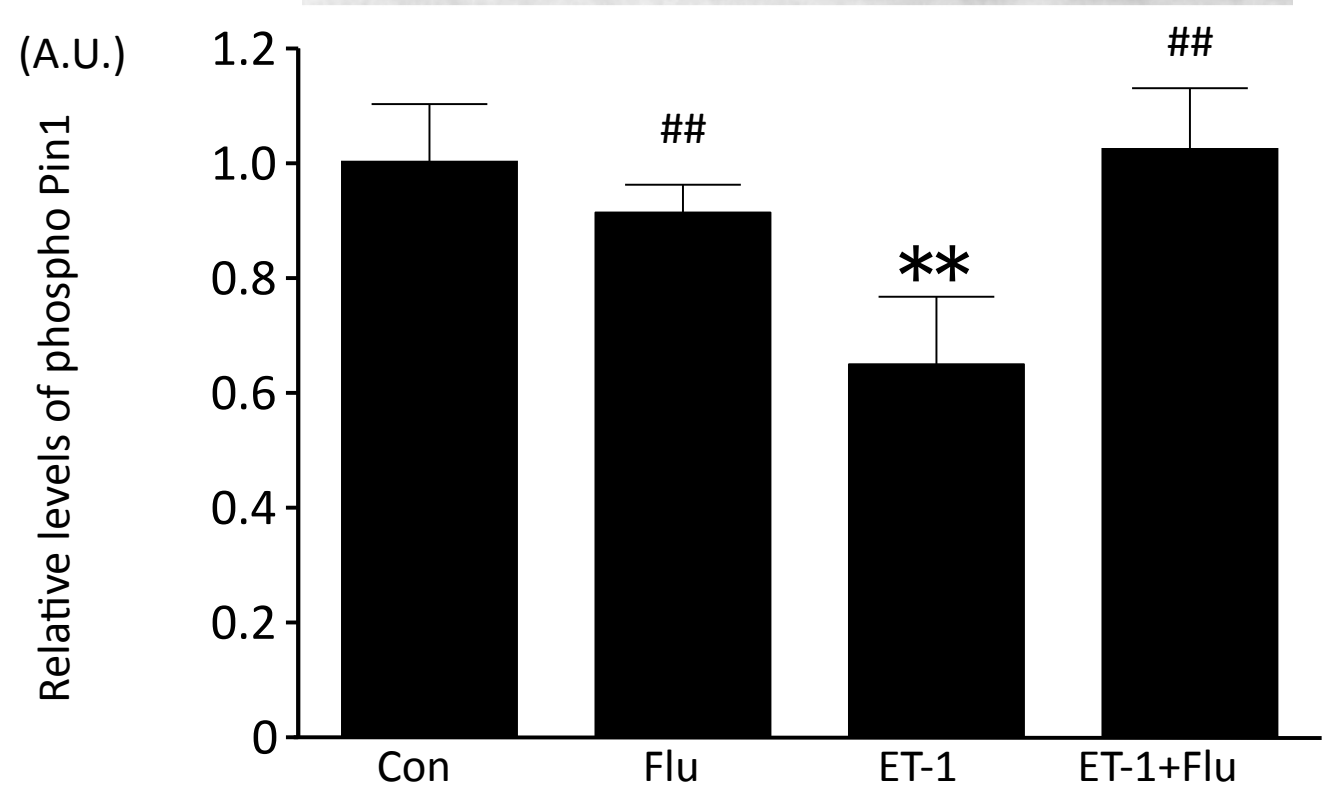


Figures 6A-6E Sakai S, et al
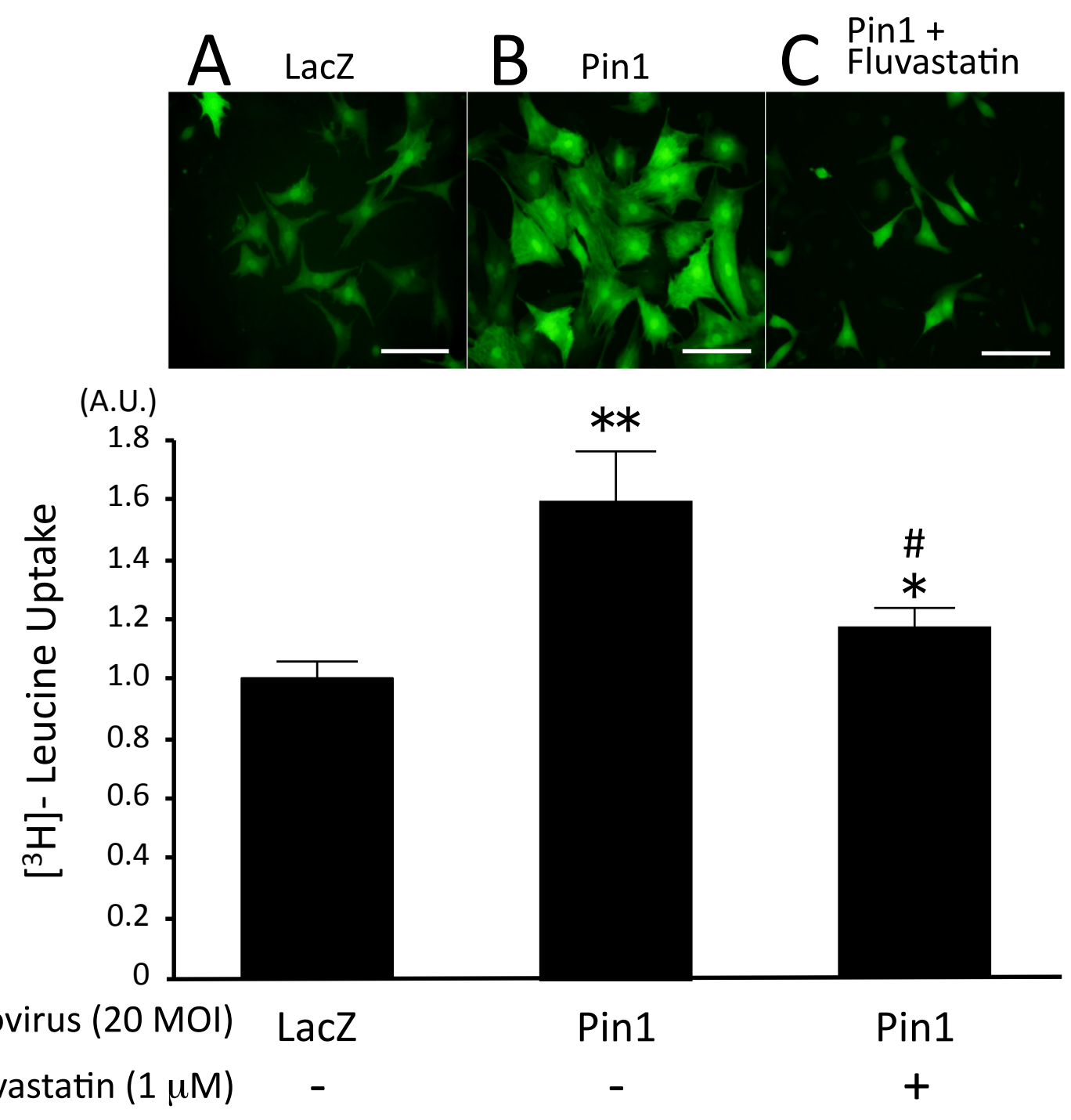

$E$

Phospho-Pin1

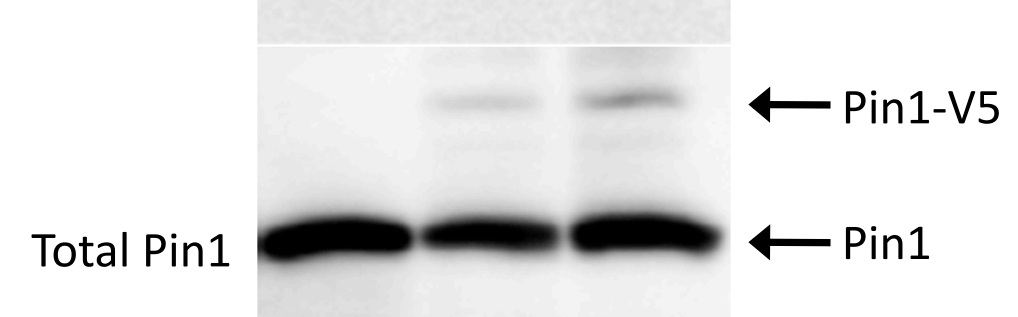

V5

$\beta$-actin

Adenovirus (20 MOI) LacZ Pin1 Pin1

Fluvastatin $(1 \mu \mathrm{M}) \quad$ -

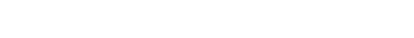

Fluvastatin $(1 \mu \mathrm{M}) \quad-\quad \quad-\quad+$ 